\title{
VERFASSUNG UND RECHT IN ÜBERSEE AUS DER SICHT DES INVESTORS
}

\begin{abstract}
Wer in Entwicklungsländern investieren möchte, errechnet nicht nur seinen möglichen Gewinn, ihn beschäftigt auch die Frage, inwieweit seine Kapitalanlage nichtkommerziellen Risiken ausgesetzt ist. Zur Abwägung derartiger Gefahren genügt es nicht, daß man dieses spannungsreiche Feld allein von der Rechtslage her analysiert. Der komplexe Charakter solcher rechnerisch nicht erfaßbaren Risiken macht es vielmehr erforderlich, die Verflechtung und gegenseitige Abhängigkeit der einschlägigen politischen, sozialen, wirtschaftlichen und rechtlichen Beziehungen des Anlagelandes zu durchleuchten ${ }^{1}$. Dabei empfiehlt es sich dringend, fachkundigen Rat einzuholen, z. B. von Außenhandelskammern, Regionalvereinen und spezialisierten Forschungsinstituten.

So wünschenswert ein stärkeres Engagement des Privatkapitals in Übersee sein mag, so erklärlich sind die Vorbehalte auf seiten der Unternehmerschaft in den Industrieländern. Namentlich die Abhängigkeit von einer fremden Rechtsordnung und der Umgang mit fremden Verwaltungsinstanzen bergen einen erhöhten Unsicherheitsfaktor. Unter Verzicht auf eine in der Fachliteratur verbreitete Kasuistik will der folgende Beitrag den Investor auf die Ursachen typischer Konfliktsituationen hinweisen und Möglichkeiten einer entsprechenden Risikominderung aufzeigen.
\end{abstract}

\section{Die Risiken und ihre Ursachen}

Die Gefahren, um die es hier geht, gehören zum Bereich vermögensentziehender Hoheitsakte und äußern sich in Enteignungen und Verstaatlichungen. Sie umfassen über den förmlichen Eigentumsentzug hinaus alle Maßnahmen, die das Wesen des Eigentums antasten und daher als enteignungsgleiche Eingriffe - auch "geräuschlose" oder "schleichende" Enteignung - bezeichnet werden. Hierzu zählen insbesondere: die Verweigerung von Importlizenzen für notwendige Ausrüstungsgüter, konfiskatorische Besteuerungen, rücksichtslose Preisstopps und weitreichende Transfer-Restriktionen. Gegen solche politischen Risiken sind Entwicklungsländer im allgemeinen weniger sicher als Industrieländer. Wer in wirtschaftlich zurückgebliebenen Ländern eine Niederlassung oder Beteiligung unterhält, begegnet einem tiefgreifenden Wandel von Wertvorstellungen und Verhaltensweisen. Die dadurch vielfach bedingte politische Instabilität bleibt nicht ohne Einfluß auf die Investitionsentscheidung des ausländischen Unternehmers. Seitdem der wirtschaftliche und soziale Aufbau in den Entwicklungsländern zunehmend politisiert wird, ist die Sicherheit des Auslandskapitals eng mit den Wechselfällen der Innenpolitik dieser Staaten verknüpft ${ }^{2}$. Ihre Wirtschaftsordnung entspricht dem jeweiligen Kräfteverhältnis von Regierung, Parteien, Gewerkschaften, militärischen Gruppen, Stammesverbänden, Religionsgemeinschaften usw. Für den potentiellen Investor ist es besonders wichtig $\mathrm{zu}$ wissen, ob die politisch-ideologische Zielsetzung des Anlagelandes stärker die Kollektividee betont oder mehr das wohlverstandene Individualinteresse berücksichtigt.

Die wirtschaftspolitische Grundhaltung einer Regierung zeigt sich in der Steuerund Importgesetzgebung, der Investitionsplanung und Rohstoffpolitik, im Kreditwesen und der Art, wie die öffentliche Hand sich wirtschaftlich betätigt. Ein erhöhtes Maß an obrigkeitlicher Lenkung findet man allerdings in allen Entwicklungsländern, ohne daß deren Wirtschaftsordnung deshalb bereits zentralistische

1 Vgl. H. G. Meissner, Das Entwicklungsgeschäft, Berlın 1966, S. $32 \mathrm{ff}$. 2 Vgl. John Agad, Der politische Faktor in den internationalen privaten Kapitalwanderungen, Genf 1960,
S. $17 \mathrm{ff}$., $122 \mathrm{ff}$. 
Züge trägt. Besonders das Auslandskapital pflegt Einschränkungen zu unterliegen, die sich aus den spezifischen Interessen der Anlageländer ergeben. So ist ihnen häufig daran gelegen, inländisches Kapital zu beteiligen, Mindestlöhne für einheimische Arbeitskräfte festzusetzen, in der Unternehmensführung mitzuentscheiden und eine angemessene Beteiligung am Gewinn zu erhalten ${ }^{3}$. Gehören Beschränkungen der genannten Art noch zum normalen Unternehmerrisiko, so ist dies bei staatlichen Interventionen mit Enteignungscharakter nicht mehr der Fall. Durch solche Eingriffe in die Kapitalsubstanz entstehen Schäden, die den zahlenmäßig kalkulierbaren, ökonomischen Gefahrenbereich überschreiten und die Ausübung der Unternehmerinitiative oft recht empfindlich stören. Nicht selten zwingen diese Maßnahmen den Investor, seine Geschäftstätigkeit in dem betreffenden Land völlig einzustellen und schwere Verluste hinzunehmen.

\section{Das Enteignungsrisiko}

Vermögensentziehende Staatseingriffe haben ihre Ursache in den besonderen Umweltbedingungen, auf die ausländisches Privatkapital in wirtschaftlich rückständigen Ländern trifft. Es begegnet dort oft Vorbehalten, ja sogar unverhohlener Ablehnung und Diskriminierung, einem Vermächtnis aus dem Kolonialzeitalter, das sich mit der bei uns üblichen ökonomischen Betrachtungsweise nur schwer erfassen läßt. Wenn sich einige Entwicklungsländer in den letzten Jahren auf diesen Standpunkt versteift haben, dann vor allem deshalb, weil sie befürchten, erneut Objekte fremder Ausbeutung und Bevormundung $\mathrm{zu}$ werden. Ihr erwachtes Nationalgefühl und eine entsprechende Propaganda haben häufig dazu geführt, daß sich Mißtrauen und Feindseligkeit gegenüber den „westlichen Imperialisten“ verbreiteten ${ }^{4}$. Die Verbitterung einer heranwachsenden Schicht einheimischer Geschäftsleute, die mit dem kapitalstarken Ausländer nicht konkurrieren können, bleibt nicht ohne Rückwirkung auf das Anlageklima. Mißhelligkeiten können auch die Folge eines unterschiedlichen Lebensstandards sein, wie er entsteht, wenn ausländische Gesellschaften bedeutend höhere Löhne und Gehälter zahlen als die entsprechenden einheimischen Unternehmen. Die Ressentiments sind um so größer, je mehr Privilegien der Investor für sich in Anspruch nimmt. Sichtbare Wettbewerbsvorteile ausländischer Firmen verletzen allzu leicht das Selbstbewußtsein der unterentwickelten Völker und sind daher geeignet, die Atmosphäre erheblich zu belasten. Auch „sozialistisches“ Ideengut, in den ehemals abhängigen Gebieten unterschiedlich stark verbreitet, kommt als mögliche Störungsquelle in Betracht ${ }^{5}$.

\section{Das Transferrisiko}

Eine Niederlassung in Übersee ist für den Investor nur sinnvoll, wenn er die erwirtschafteten Gewinne ungehindert in sein Heimatland transferieren kann. Transferbeschränkungen können den Geschäftsinhaber wie eine förmliche Enteignung treffen; denn zum Wesen des Eigentums gehört grundsätzlich die freie Verfügungsbefugnis des Berechtigten ${ }^{6}$. Unterentwickelte Länder, die gelegentlich in dieser Weise intervenieren, bringen gern das Argument, ausländische Privatinvestitionen hätten auf ihre Wirtschaft einen entkapitalisierenden Effekt. In der Tat sind ihre Devisenabflüsse für den Rücktransfer von Kapitalien, Gewinnen, Dividenden und Lizenzgebühren zeitweise wesentlich höher als der Devisenzugang

\footnotetext{
3 Vgl. Helmut Giesecke, Industrieinvestitionen in Entwicklungsländern - Risiken und Chancen, Hamburg 1963, S. $40 \mathrm{ff}$.

$4 \mathrm{Vgl}$. Dieter Weiss, Die Unternehmung bei wachsender Integration der Märkte, in: Vorträge des 18. Deutschen Betriebswirtschafter-Tages, Berlin 1965, S. 320.

5 Vgl. Oswald Hahn, Probleme der Kapitalerhaltung in Entwicklungsländern, in: Vorträge des 19. Deutschen Betriebswirtschafter-Tages, Berlin 1966, S. 219.

6 Vgl. im einzelnen The National Industrial Conference Board, Obstacles and Incentives to Private Foreign Investors, New York 1965.
} 
für Neuinvestitionen. Es wäre jedoch verfehlt, wollte man hieraus mit einigen Entwicklungsländern den Schluß ziehen, unbeschränkte Gewinnüberweisungen würden ihre ohnehin schwierige Zahlungsbilanzsituation noch verschlechtern. Im Gegenteil, produktiv angelegtes Auslandskapital trägt dazu bei, die Zahlungsbilanz dieser Länder zu entlasten; denn es dient der Herstellung von Gütern, die entweder exportiert werden oder den Import ersetzen. Auslandsfirmen, denen angemessene Bedingungen eingeräumt werden, neigen außerdem dazu, einen großen Teil ihrer Reingewinne über längere Zeit wieder zu investieren, also im Anlageland zu belassen. Hinzu kommen die positiven Auswirkungen auf die einheimische Wirtschaft ganz allgemein. So liefert der ausländische Unternehmer nicht nur Kapital, sondern zugleich technisches und organisatorisches Wissen, woran es den Entwicklungsländern besonders fehlt. Bei exportorientierten Industriebetrieben kommt dem Land überdies die internationale Geschäftserfahrung des Investors zugute$^{7}$. Schließlich erreichen die Kapitalimportländer mit einer strengen Devisenbewirtschaftung gerade das Gegenteil dessen, was sie beabsichtigen. Der Unternehmer, der sich normalerweise mit periodischen Gewinntransferierungen begnügen würde, sieht sich unter diesen Umständen genötigt, seine gesamte Investition rückgängig zu machen. Eine solche Kapitalflucht wird, wie hieraus ersichtlich, nicht von der Rendite bestimmt, sondern von Erwägungen der Sicherheit gegen politische Risiken.

\section{Administrative Risiken}

Manche Einbuße erleidet der Investor durch den zermürbenden Kampf mit der Bürokratie. In den Entwicklungsländern reicht der Arm der Verwaltung viel weiter als bei uns. Auffallend sind dort oft eine lange Bearbeitungsdauer, mangelnde Sachkenntnis, Desinteresse und eine Scheu vor Verantwortung. Die Schwerfälligkeit der Behördenpraxis wird durch die Einschaltung zahlreicher Kontrollinstanzen noch erhöht. Man hält dieses System jedoch für unentbehrlich, um die passive Bestechung zu bekämpfen, der gegenüber der Staatsdiener eines unterentwickelten Landes im allgemeinen anfälliger ist als sein Amtskollege in den Industrieländern. Durch eine ihm günstige Anlagegesetzgebung darf ein Auslandsinvestor sich also nicht zu der Annahme verleiten lassen, dadurch allein sei bereits eine reibungslose Zusammenarbeit mit der Verwaltung gewährleistet. Auf der anderen Seite begegnet man immer wieder der Tatsache, daß ein in Übersee tätiger Europäer die dortige Bürokratie wegen einer Maßnahme, die er daheim widerspruchslos hingenommen hätte, vorschnell als unternehmer- und ausländerfeindlich verurteilt. Er sollte sich stets fragen, ob ein vermeintlicher Mangel der Administration nicht in Wahrheit auf sein eigenes Verhalten zurückzuführen ist ${ }^{8}$. Die betreffenden Länder scheinen sich auch selbst mehr und mehr der Bedeutung bewußt zu werden, die einer gestrafften Behördentätigkeit bei ihrem wirtschaftlichen Aufbau zukommt.

\section{Möglichkeiten einer Risikominderung}

Der Investor hat es in der Hand, seine Sicherheit zu erhöhen, indem er sich fester in die Wirtschaft des Anlagelandes einfügt. Zweckdienlich ist ein Verhalten, das den Vorstellungen und Erwartungen der Gastgeber entgegenkommt, ohne damit den privatwirtschaftlichen Sinn der Kapitalanlage auszuhöhlen. Dies setzt ein starkes Einfühlungsvermögen und dementsprechend gründliche Landeskenntnisse voraus. Sorgfältig gepflegte Kontakte zur Bürokratie und ein freundschaftliches Verhältnis zu einflußreichen Persönlichkeiten sind dabei überaus nützlich. Die

\footnotetext{
7 Burke Knapp, Interview mit der Banque Marocaine du Commerce Extérieur, Monthly Bulletin of Information, Casablanca $1965, \mathrm{Nr} .59$.

8 Vgl. Oswald Hahn, a. a. O., S. 212 f.; Dieter Weiss, a. a. O., S. 328.
} 
Furcht vor einer Überfremdung läßt sich in vielen Fällen dadurch beseitigen, daß der Ausländer inländisches Kapital beteiligt und einheimische Kräfte in die Geschäftsleitung aufnimmt. Diese Art der Zusammenarbeit bringt allerdings ein fremdes Element in die Unternehmensführung, das um so störender sein kann, je weniger in dem betreffenden Land nach modernen Grundsätzen gearbeitet und rentabel kalkuliert wird. Doch läßt sich dieses Risiko bei einiger Umsicht in Grenzen halten.

Freilich gewährleistet auch das behutsamste Vorgehen nicht immer genügende Sicherheit. Wirksame institutionelle Garantien müssen hinzukommen. Ein Land, das sich um Wirtschaftshilfe in Form ausländischen Privatkapitals bemüht, wird daher den gewünschten Erfolg nur haben, wenn es dem Kapitaleigner ausreichenden gesetzlichen und vertraglichen Schutz gewährt ${ }^{9}$. Aus diesem Grunde haben mehr als 30 Entwicklungsländer standardisierte Investitionsförderungsverträge mit der Bundesrepublik Deutschland abgeschlossen ${ }^{10}$. Mit ihren weitreichenden Schutzbestimmungen tragen die Abkommen wesentlich dazu bei, eine den zwischenstaatlichen Kapitalfluß belebende Atmosphäre gegenseitigen Vertrauens zu schaffen ${ }^{11}$. Wirkungsvoll ergänzt werden die Vertragsregeln durch ein staatliches Garantiesystem, das dem deutschen Unternehmer die Möglichkeit eröffnet, Verluste an einer förderungswürdigen Auslandsanlage, die durch politische Ereignisse im Anlageland entstehen, ähnlich wie bei einer Versicherung abdecken zu lassen ${ }^{12}$.

GÜNTER WIEDENSOHLER

9 Vgl. A. A. Fatouros, Government Guarantees to Foreign Investors, New York - London 1962, S. 358 ff.

$10 \mathrm{Vgl}$ den Vertrag zwischen der Bundesrepublik Deutschland und der Republik Tunesien über die Förderung und den gegenseitigen Schutz von Kapitalanlagen, Bundesgesetzblatt 1965, II, S. 1377 ff.

11 Vgl. Rolf Berger, Der Schutz deutscher Kapitalanlagen im Ausland durch Investitionsförderungsverträge, in: Der Betrieb 1965, Heft 16, S. 585.

12 Siehe im einzelnen die Rid̆tlinien für die Ubernahme von Bundesgarantien für Kapitalanlagen im Ausland vom 13. Januar 1967, Bundesanzeiger, 18. Januar 1967. 